AN. MED. INTERNA (Madrid) Vol. 18, N. $^{\circ} 6$, pp. 305-308, 2001

\title{
Monitorización ambulatoria de presión arterial (MAPA) en enfermos jóvenes
}

\author{
N. R. ROBLES, M.I. DOMÍNGUEZ TRISTANCHO, B. CANCHO, E. SÁNCHEZ \\ CASADO \\ Unidad de Hipertensión Arterial. Sección de Nefrología. Hospital Infanta Cristina. \\ Badajoz
}

\author{
AMBULATORY BLOOD PRESSURE MONITORING (ABPM) IN \\ YOUNG PATIENTS
}

\section{RESUMEN}

Objetivo: Aunque se han definido diversas indicaciones genéricas para el uso de la MAPA, sus indicaciones específicas en grupos de población especiales no han sido completamente definidos. Este es el caso de su utilidad en el diagnóstico en pacientes jóvenes, es decir, aquellos que tiene menos de 30 años.

Métodos: Se ha realizado MAPA en 52 casos consecutivos de HTA en pacientes menores de 30 años (edad media $23,4 \pm 4,9$, entre 12 y 30 años). El estudio se realizó en todos los casos sin tratamiento (si fue preciso suspenderlo, esta suspensión se realizó al menos 10 diez días antes) según los criterios habituales (colocación en brazo no dominante, definición de periodo diurno y nocturno personalizada, mediciones cada 15 min. en el periodo diurno y cada $20 \mathrm{~min}$. en el nocturno). Se definió normotensión como un promedio de PA inferior a $130 / 80 \mathrm{mmHg}$ en $24 \mathrm{~h}$. La reacción de bata blanca (RBB) se calcula como $1^{\mathrm{a}}$ medición/media periodo diurno. Se consideró la presencia de RBB como un valor superior a 1,1. Para valorar la normalidad del descenso nocturno se consideró una reducción mínima del $10 \%$ respecto a los valores diurnos.

Resultados: En 37 (71\%) casos se comprobó que el paciente presentaba normotensión en la MAPA. No hubo diferencias en la edad entre normotensos $(23,9 \pm 5,1)$ e hipertensos $(23,2 \pm 4,0$ años) ni en el sexo (normotensos 21 varones y 16 mujeres, hipertensos 10 varones y 3 mujeres). La PA media de los normotensos fue 119/72 ( $<<0,001$ frente a hipertensos, $135 / 89 \mathrm{mmHg}$ ). No hubo diferencias en el descenso nocturno entre ambos grupos. La RBB fue más intensa en el grupo de normotensos $(1,17 \pm 0,12)$ frente a hipertensos $(1,04 \pm 0,08, \mathrm{p}<0,001)$, aunque 4 hipertensos presentaban RBB $(1,11 \pm 0,05)$.

Conclusiones: La realización de MAPA para aconsejable para confirmar el diagnóstico de HTA en los pacientes menores de 30 años antes de realizar otras técnicas diagnósticas.

PALABRAS CLAVE: Hipertensión arterial. Joven. Registro ambulatorio de presión arterial.

\section{ABSTRACT}

Objective: To defining the criteria for performing ambulatory blood pressure monitoring $(A B P M)$ in young patients.

Method: It is reported the experience with $A B P M$ on 52 consecutive patients (younger than 30 years old) consulting for hypertension (mean age $23.4 \pm 4.9$ years). The ambulatory $B P$ was measured noninvasively for twenty-seven hours by the Spacelabs 90207 device programmed to measure BP every fifteen minutes during daytime and every 20 minutes during nighttime. The definition of daytime and nighttime was made on the basis of wakefulness and sleep or bed rest periods, obtained from a diary kept by the subject, normal nocturnal BP drop was defined as a decrease higher of $10 \%$ versus daytime values. It was defined normal BP an 24 hours ambulatory $B P<130 / 80 \mathrm{mmHg}$.

Results: Thirty seven patients (71\%) were normotensives. There were not differences between normotensive and hypertensive patients neither by age (normotensive $23.9 \pm 12.5$, hypertensive $23.3 \pm 4.0$ years), nor by sex (normotensive, 21 men and 16 women; hypertensive, 10 men and 3 women). Mean $24 \mathrm{~h}$ BP of normotensive patients was 119/72 $\mathrm{mmHg}(p<0.001 \mathrm{vs}$. hypertensive, $135 / 89 \mathrm{mmHg})$. There were not diffe rences in nocturnal BP drop. White-coat reaction was more intense in normotensive patients $(1.17 \pm 0.12$, vs. hypertensive $1.04 \pm 0.08, p<$ $0.001)$. Four hypertensives showed white-coat reaction (1.11 \pm 0.05$)$.

Conclusion: ABPM is a helpful diagnostic tool in young patients. It should be routinely performed as first exploration in all patients younger than 30 years consulting for hypertension.

KEY WORDS: Hypertension. Young. Blood pressure monitoring.

Robles NR, Domínguez Tristancho MI, Cancho B, Sánchez Casado E. Monitorización ambulatoria de presión arterial (MAPA) en enfermos jóvenes. An Med Interna (Madrid) 2001; 18: 305-308.

\section{INTRODUCCIÓN}

La MAPA es un elemento diagnóstico de extraordinario interés para la enfermedad más común que podemos encontrar en nuestra sociedad. Su uso se ha generalizado progresivamente en la práctica clínica tanto general como especializada. Además de sus utilidades desde el punto de vista experimental y para la investigación clínica $(1,2)$, se han definido dos usos esenciales para la técnica. El primero de ellos es la confirmación del diagnóstico de HTA (o dicho al revés, la negación del diagnóstico

Trabajo aceptado: 25 de Enero de 2001

Correspondencia: Nicolás Roberto Robles Pérez. Sección de Nefrología. Hospital Infanta Cristina. Ctra. de Portugal s/n. 06080. Badajoz. 
de hipertensión de bata blanca o hipertensión clínica aislada). El segundo es la valoración de la efectividad del tratamiento utilizado tanto desde el punto de vista de la intensidad de la respuesta hipotensora, como desde el punto de vista de la persistencia del efecto hipotensor durante las 24 horas del día (3-5).

En estas condiciones sería probablemente ideal que todos los enfermos que debutan con hipertensión arterial fueran estudiados con MAPA antes de indicar tratamiento hipotensor. Teóricamente, esto permitiría evitar hasta un $20 \%$ de tratamientos antihipertensivos innecesarios (6). Sin embargo, la disponibilidad actual (y a medio plazo) de este tipo de equipos y la enorme masa de población susceptible de ser estudiada (7) obligan a intentar definir unos criterios mínimos de selección de las personas en las que la presión arterial ambulatoria debe ser examinada.

En este estudio se ha valorado la utilidad de la MAPA en las personas jóvenes que consultan por presentar hipertensión arterial antes de iniciar estudios y/o tratamiento de la enfermedad hipertensiva.

\section{PACIENTES Y MÉTODOS}

Se incluyeron en el estudio 52 pacientes estudiados consecutivamente, con una edad media de 23,4 44,9 años (12-30 años), siendo 32 hombres y 20 mujeres (la figura 1 presenta la distribución por edades). Ninguno de los pacientes recibía tratamiento en el momento de realizar la MAPA pero todos habían mostrado cifras de presión arterial elevada en consulta (> 140/90 $\mathrm{mmHg}$ ) de forma repetida.

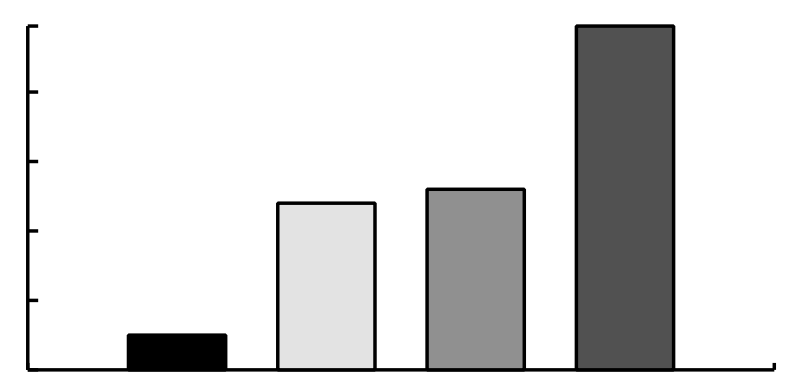

Fig. 1. Distribución por edades de los enfermos incluidos en el estudio.

En todos los casos se realizó un RAPA de 24 horas de duración utilizando un monitor Spacelab 90207 programado para efectuar tomas de presión arterial cada 15 minutos en el periodo diurno y cada 15-20 minutos en el periodo nocturno. La definición de los periodos diurno y nocturno fue variable en cada paciente dependiendo del diario de actividad de este. El aparato se colocó en todos los casos en el brazo no dominante. Se calcularon los valores medios de la PAS y PAD, los índices de carga sistólica (ICS) y diastólica (ICD), el descenso nocturno de la PA (expresado como cociente [PA diurna - PA nocturna] * 100/PA diurna) tanto para valores sistólicos como diastólicos y la reacción de alerta (expresada como cociente primera lectura/promedio del periodo diurno) también para valores sistólicos y diastólicos.
Se consideró enfermo hipertenso (por tanto, necesitado de iniciar tratamiento) un promedio de PA de 24 horas $>135 / 85$ $\mathrm{mmHg}$ (o bien > 140/90 $\mathrm{mmHg}$ en el periodo diurno o > $120 / 80$ en el periodo nocturno) (8). Los pacientes con PA > $130 / 80 \mathrm{mmHg}$ en el promedio de 24 horas se consideraron limítrofes con la hipertensión arterial (8). Para la definición de ritmo nictameral alterado, se consideró un descenso nocturno de la PA sistólica o diastólica inferior al $10 \%$ de los valores diurnos. De la misma manera, se definió la presencia de reacción de bata blanca cuando el cociente primera medición/media del periodo diurno era $>1,1$.

En todos los pacientes se realizó exploración física completa, tras lo cual se obtuvieron muestras para determinar bioquímica sanguínea (incluyendo parámetros de función renal e iones), hemograma, análisis de orina (con ionograma) y microalbuminuria. También se realizó examen de fondo de ojo, radiografía simple de tórax posteroanterior y lateral, ECG y ecografía con estudio con modo Doppler de la vascularización renal. En los casos que presentaban hipertensión se añadieron estudios hormonales (actividad renina plasmática, aldosterona, tiroxina y TSH plasmática, cortisol y catecolaminas urinarias) y TAC helicoidal con reconstrucción de las arterias renales.

Las variables continuas han sido expresadas como media \pm desviación estándar. La comparación de estos valores se realizó mediante la prueba de la t de Student para muestras independientes. Las variables de frecuencia fueron comparadas mediante la prueba de Chi cuadrado y la prueba exacta de Fischer cuando el número de datos era igual o inferior a 5 en algunas de las categorías comparadas. El estudio estadístico de los datos se realizó utilizando el programa informático Sigma (Madrid, Horus Informática).

\section{RESULTADOS}

En total $13(25,0 \%)$ enfermos eran hipertensos según los criterios de la MAPA, de ellos, 4 presentaban HTA sistólica aislada. En el estudio posterior de HTA secundaria, 11 casos fueron considerados esenciales, 1 caso se debía a HTA vasculorrenal y 1 a glomerulonefritis crónica. La distribución por segmentos de edad de los hipertensos se muestra en la figura 2. Treinta y siete enfermos fueron diagnosticados de hipertensión

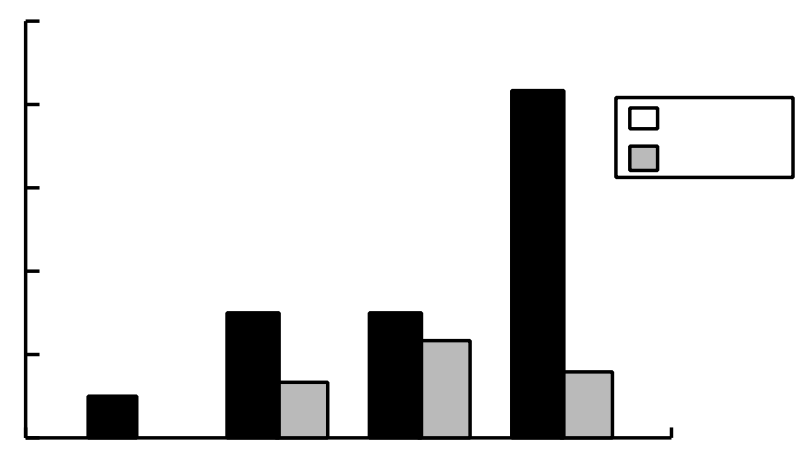

Fig. 2. No hubo diferencias en la incidencia de hipertensión real entre los grupos de edad. El diagnóstico de hipertensión de bata blanca no fue menos frecuente en los pacientes de mayor edad. 
clínica aislada, es decir la MAPA mostraba valores de normotensión y las exploraciones realizadas no demostraron ninguna alteración relacionada con la HTA bien como causa, bien como consecuencia. Dos pacientes mostraban valores límite de PA (un hombre y una mujer, edad media 16,5 $\pm 0,7$ años, el tamaño la muestra no permite hacer comparaciones estadísticas).

No hubo diferencias entre hipertensos y normotensos por edad (normotensos 26,8 $\pm 5,6$, hipertensos 23,3 $\pm 7,6$ años) ni por sexo (prueba de Chi cuadrado, representado en figura 3 ). Los pacientes normotensos tendían a ser más obesos que los hipertensos según el índice de masa corporal sin alcanzar significación estadística (normotensos 26,8 $\pm 5,6$, hipertensos $23,3 \pm 7,6, \mathrm{p}<0,1)$. Los edad de los pacientes con HTA sistólica también se acercaba a la significación estadística sin alcanzarla (edad 20,3 $\pm 3,4$ años, p $<0,1)$.

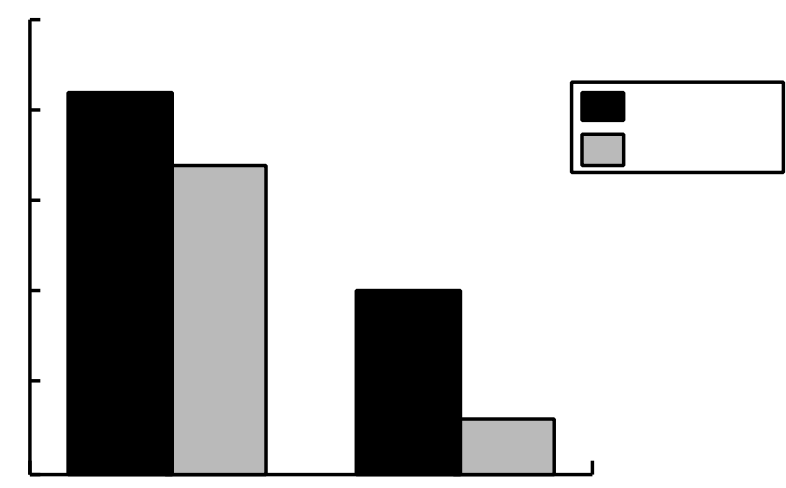

Fig. 3. No se apreciaron diferencias de sexo entre normotensos e hipertensos.

La media de PAS en $24 \mathrm{~h}$ fue significativamente mayor en los hipertensos $(135,3 \pm 7,3 \mathrm{mmHg})$ que en los normotensos $(118,9 \pm 9,6 \mathrm{mmHg}, \mathrm{p}<0,001)$. La PAD promedio en $24 \mathrm{~h}$ también fue significativamente mayor en los hipertensos $(89,2 \pm 8,1$ $\mathrm{mmHg}$ ) que en los normotensos $(71,8 \pm 5,9 \mathrm{mmHg}, \mathrm{p}<0,001)$. No hubo diferencias en la PAS en consulta (hipertensos $145,6 \pm 11,6$, normotensos 143,6 $\pm 14,4 \mathrm{mmHg}$ ). La PAD en consulta mostraba una diferencia que no llega a alcanzar significación estadística (hipertensos 99,5 $\pm 8,1$, normotensos $92,7 \pm 11,8$ $\mathrm{mmHg}, \mathrm{p}<0,1)$. El número de enfermos que presentaban PAD superior a $100 \mathrm{mmHg}$ en consulta también se acercaba a la significación estadística (hipertensos $\mathrm{n}=7,63,6 \%$; normotensos $\mathrm{n}=11,27,5 \%, \mathrm{p}=0,054$, prueba de Fischer).

El fenómeno de bata blanca era más intenso en los normotensos tanto para la PAS (normotensos, $1,17 \pm 0,12$, hipertensos, 1,04 $\pm 0,08, \mathrm{p}<0,001$ ), como para la PAD (normotensos, $1,23 \pm 0,16$, hipertensos $1,09 \pm 0,06, p<0,001)$. Treinta y cuatro enfermos normotensos y 4 hipertensos presentaban reacción de bata blanca.

No se detectaron diferencias en el descenso nocturno de la PA entre los dos grupos, ni en la PAS (normotensos 11,0 $\pm 6,1$, hipertensos 9,6 $\pm 6,7 \%$ ) ni en la PAD (normotensos 17,8 $\pm 7,4$, hipertensos $15,2 \pm 8,4 \%$ ). Desde un punto de vista cualitativo, 4 enfermos normotensos y 3 hipertensos presentaban descenso nocturno alterado, pero esta diferencia no fue significativa (prueba de Fischer).

\section{DISCUSIÓN}

El uso de MAPA permitió detectar un porcentaje importante de enfermos jóvenes que, pese a haber resultado hipertensos en las mediciones realizadas en consulta, eran normotensos, es decir, padecían hipertensión de bata blanca o hipertensión clínica aislada. Está es una cifra muy superior al porcentaje detectable de hipertensión de bata blanca, que está alrededor del $20 \%$ en los estudios conocidos realizados en población general de todas las edades (6). Es evidente la diferencia de muestra utilizada (población general frente a pacientes remitidos a una unidad hospitalaria de HTA) y esto explica fácilmente las diferencias, puesto que si bien todos los enfermos menores de 30 años que presentan cifras de PA elevadas en consulta deben ser remitidos inmediatamente a una unidad de HTA, solamente casos muy seleccionados de los pacientes de edades mayores tienen criterios de derivación.

No fue posible detectar ninguna diferencia en el sexo entre ambos grupos, pese a que existen estudios que sugieren que la hipertensión de bata blanca podría ser más frecuente en las mujeres (9-11). Tampoco fue posible hallar diferencias por edad, es decir, en el intervalo de edad estudiado no hubo un incremento del número de casos de hipertensión arterial en el segmento de los enfermos de mayor edad relativa. Este hallazgo, interesante en si mismo, puede ser atribuible a la estrecha banda de edades estudiada. Puesto que la incidencia de reacción de bata blanca parece ser similar en todas las edades $(12,13)$ es evidente el sesgo ocasionado por la edad de los pacientes en el criterio de derivación a la consulta especializada.

La disponibilidad de la MAPA ha permitido conocer la reacción de bata blanca, es decir la elevación de la presión arterial secundaria a la reacción de alerta ocasionada por la misma toma de la presión arterial, tanto cuando la toma un médico como cuando la toma el personal de enfermería (1416). La presencia de discordancias entre mediciones de PA realizadas en consultorio y mediciones efectuadas en casa o fuera del consultorio en algunos pacientes es un tema comentado desde hace tiempo en la literatura científica (17). Sin embargo su descripción específica es relativamente reciente $(14,15)$.

Es importante recordar que el fenómeno de bata blanca no excluye la existencia de hipertensión, puesto que un porcentaje significativo de hipertensos lo presentan (14). Esto lo diferencia del concepto de hipertensión de bata blanca acuñado sobre la existencia de tomas de presión arterial elevada en el consultorio médico en pacientes que presenta un MAPA diagnóstico de normotensión arterial $(6,18)$. Dentro de este grupo de pacientes se podrían incluir aquellos que presentan hipertensión arterial aislada en momentos generalmente asociados a estrés no relacionado con la presencia del médico y cuyo MAPA sería normal. Por ello se ha sugerido el uso de la denominación alternativa de hipertensión clínica aislada (19).

El fenómeno de bata blanca puede tener consecuencias importantes en la decisión terapéutica, puesto que conlleva la posibilidad de iatrogenia secundaria a sobretratamiento en pacientes aparentemente hipertensos o rebeldes al tratamiento hipotensor que están recibiendo. Los datos obtenidos sugieren que esta posibilidad debería ser valorada antes de iniciar tratamiento en todos los pacientes con edad inferior a 30 años que debuten con hipertensión arterial. Es más, la realización de la MAPA permitiría evitar la realización de un número importante de exploraciones diagnósticas que se consideran indica- 
das en estos casos, en busca de una causa curable para una hipertensión que realmente no existe. La consecuencia inmediata es que la realización protocolizada de MAPA permitiría una reducción importante de costes tanto en diagnóstico como en tratamiento en todos los enfermos jóvenes (menores de 30 años).

El uso de la MAPA presenta diversos problemas en su uso clínico. Uno de ellos reside en el limitado número de equipos disponibles y de personal suficientemente entrenado para programarlo e interpretarlo. Aunque este número es creciente, será aún difícil en algún tiempo que exista una disponibilidad universal de la técnica y todavía más lejano que la oferta supere a la demanda de este tipo de exploraciones. Por otra parte, no se debe olvidar que, aunque las complicaciones descritas con el uso de la MAPA son escasas y sin apenas gravedad desde el punto de vista clínico, la exploración es molesta y engorrosa para el enfermo que es sometida a ella.

Por todo lo expuesto, la indicación de realización de MAPA en todos los nuevos diagnósticos de hipertensión arterial no parece ni posible, ni tan siquiera deseable desde el punto de vista del enfermo, pese a su interés clínico y experimental. Es por ello preciso definir los criterios que aconsejan que la técnica sea indicada y uno de los criterios posibles es la existencia de poblaciones más o menos proclives a la presencia de hipertensión. Puesto que existe una clara correlación entre la edad de la población y la prevalencia de hipertensión arterial, parece que, como se ha valorado en este estudio, una de las poblaciones sospechosas de hipertensión de bata blanca serían los enfermos más jóvenes, donde la prevalencia es más baja. Los resultados de nuestro estudio demuestran la validez de la hipótesis: la prevalencia de hipertensión arterial real en los enfermos menores de 30 años es tan baja que justifica la confirmación del diagnóstico mediante MAPA en todos los pacientes identificados como hipertensos mediante tomas casuales.

Otra técnica utilizada en los pacientes con sospecha de hipertensión de bata blanca es la automedición de la presión arterial (AMPA). La AMPA ha demostrado ser también un buen predictor de la morbimortalidad asociada a HTA con mejor correlación que con las mediciones obtenidas mediante tomas casuales en consulta. Es indudable que la AMPA, puede tener una utilidad cierta en estos casos, especialmente como método para el despistaje, pero el diagnóstico definitivo de hipertensión de bata blanca exige la realización de MAPA, cuyos resultados muestran aún mejor correlación con la existencia de daño de órganos diana (20).

La MAPA es un elemento de extraordinaria utilidad en los enfermos hipertensos. Su uso ha permitido definir dos situaciones especiales dentro de estos enfermos, la alteración del descenso nocturno de la PA y la hipertensión de bata blanca. Su disponibilidad limitada obliga a contemplar las condiciones ideales para indicar su realización. La hipertensión de bata blanca, a la vista de estos resultados, es una situación frecuente en los enfermos menores de 30 años que consultan por hipertensión arterial detectada mediante tomas casuales. Parece conveniente recomendar el uso de la MAPA, como elemento diagnóstico de amplio interés, en los pacientes jóvenes.

\section{Bibliografía}

1. Coats AJS, Conway J, Somers VK, Isea JE, Sleight P. Ambulatory pressure monitoring in the assessment de antihypertensive therapy. Cardiovasc. Drugs Ther 1989; 3: 303-311.

2. Waeber B, Heynen G, Brunner HR. Analysis of ambulatory blood pressure monitoring: the problem of "white-coat" hypertension, responders and non-responders. Blood Press Monit 1996; 1: 289-291.

3. Redon J, Campos C, Narciso MI, Rodicio JI, Pascual JM, Ruilope LM. Prognostic value of ambulatory blood pressure monitoring in refractrory hypertension. A prospective study. Hypertension 1998; 31: 712-718.

4. Mora J, Ocon J. Presión arterial casual frente a registro continuo de presión arterial ambulatoria. Rev Clin Esp 1991; 189: 368-373.

5. White B, Morganroth J. Usefulness of ambulatory monitoring of blood pressure in assessing antihypertensive therapy. Am J Cardiol 1989; 63: 94-98.

6. Pickering TG, James GD, Boddie C, Harshfield GA, Blank S, Laragh JH. How common is white coat hypertension. JAMA 1988; 259: 225-228.

7. Banegas JR, Villar F, Pérez C, Jiménez R, Gil E, Muñiz J, et al. Estudio epidemiológico de los factores de riesgo cardiovascular en la población española de 35 a 64 años. Rev San Hig Pub 1993; 67: 419-455.

8. Pickering T. for the American Society of Hypertension Ad Hoc Panel. Recommendations for the use of Home (Self) and Ambulatory Blood Pressure Monitoring. Am J Hypertens1996; 9: 1-12.

9. James GD, Marion R, Pickering TG. White-coat hypertension and Monit 1998; 3: 281-287.

10. Hernández del Rey P, Armario P, Sánchez P, Castesague J, Pont F. Frecuencia de la hipertensión de bata blanca en la hipertensión leve. Perfil de riesgo cardiovascular y afectación orgánica temprana. Med Clin (Barc.) 1996; 106: 690-694.

11. Martínez MA, García-Puig J, Martín JC, Guallar-Castillón P, Aguirre de Carcer A, Torre A, et al. Frecuency and determinants of white coat hypertension in mild to moderate hypertension: a primary care-based

study. Am J Hypertens 1999; 12: 251-259.

12. Mayoral E, Lapetra J, Santos JM, Lopez A, Ruiz J, Cayuela A. El efecto de bata blanca en atención primaria. Análisis en los pacientes con hipertensión arterial de nuevo diagnóstico. Med Clin (Barc.) 1997; 108: 485 489.

13. Mancia G, Sega R, Bravi C, De Vito G, Valagussa F, Cesana G et al. Ambulatory blood pressure normality: results from the PAMELA study. J Hypertens 1995; 12: 1377-1390.

14. Mancia G, Bertinieri G, Grassi G, Parati G, Promidossi G, Ferrari AU, et al. Effect of blood pressure measurements by the doctor on patient's blood pressure and heart rate. Lancet 1983; ii: 695-697.

15. Mancia G, Parati G, Promidossi G, Grassi G, Casadei R, Zanchetti A. Alerting reaction and rise in blood pressure during measurements by physician and nurse. Hypertension 1987; 9: 209-215.

16. Shimada K, Ogura H, Kawamoto K, Ishida H, Ozana T. Non invasive ambulatory blood pressure monitoring during clinic visit in elderly patients. Clin Exp Hypertens (A) 1990; 2: 151-170.

17. Ayman D, Goldshine AD. Blood pressure determinations by patients with essential hypertension: the difference between clinic and home reading before treatment. Am J Med Sci 1940; 200: 465-470.

18. Hoegholm A, Kristensen KS, Madsen NH, Svedsen TL. White coat hypertension diagnosed by 24-h ambulatory monitoring . Examination of 159 newly diagnosed hypertensive patients. Am J Hypertens 1992; 5: 64-70.

19. Mancia G, Zanchetti A. White-coat hypertension: innappropiate names, mistaken concepts and misunderstandings. What should we do from now on? J Hypertension 1996.

20. Stergiou CS, Zourbaki AS, Skeva II, Mountokalakis TD. White coat effect detected using self-monitoring of blood pressure at home. Comparison with ambulatory blood pressure. Am J Hypertens 1998; 11: 820-827. 